\title{
An Interplanetary Magnetic Field Dependent Model of the Ionospheric Convection Electric Field
}

\author{
J. J. SOJKa, C. E. Rasmussen, AND R. W. SChunK \\ Center for Atmospheric and Space Sciences, Utah State University, Logan
}

\begin{abstract}
A mathematical model of the magnetospheric electric field imposed upon the ionosphere is presented. The model provides an interplanetary magnetic field (IMF) dependent description of the magnetospheric electric field at ionospheric altitudes for global ionospheric and thermospheric modelers. Although many theoretical and empirical ionospheric convection models have been published, none give both a quantitative and a general $K p$, IMF $\left(B_{x}, B_{y}, B_{z}\right)$ dependent description. The need for such a model is particularly pressing with the success of the Dynamic Explorer (DE) mission. As a result of this mission, extensive data sets of thermospheric and ionospheric parameters for well-defined IMF orientations have been acquired. These data sets, especially for northward IMF orientations, are so complex that global models of both the thermosphere and ionosphere are required to help unravel the physics. Our convection model is an empirical model based upon a synthesis of published observations and current understanding. It describes the convection electric field by a set of simple functions which are dependent on $K p$ and IMF $\left(B_{x}, B_{y}, B_{z}\right)$. These simple functions create a framework by which current and future empirical data can be used to adjust the model. Since no recent major statistical surveys have been made of ionospheric electric fields, these functions (based upon a few published case studies) are exceedingly simple.
\end{abstract}

\section{INTRODUCTION}

In this paper we present a mathematical model of the magnetospheric electric field imposed upon the ionosphere. The model provides an interplanetary magnetic field (IMF) dependent description of the magnetospheric electric field at ionospheric altitudes for global ionospheric and thermospheric modelers. Large-scale ionospheric and thermospheric modelers require a description of the magnetospheric electric field (convection field), since it is the main transport mechanism for plasma and a major momentum and heat source for the neutral thermosphere. However, a convection model must be both quantitative as well as computationally fast; that is, as an input function it should use a relatively small amount of computer time. These two criteria were used in the construction of our convection model.

Although many theoretical and empirical models of the magnetospheric electric field have been published, none go beyond a qualitative IMF dependence or a description of the electric field for a particular IMF orientation. Our model is the result of a comparison of both published observations and qualitative models. A by-product of this study is a list of observations and data reductions which we feel are needed to define the temporal and spatial dependencies of the magnetospheric electric field on the IMF. In the conclusion, we critically discuss the validity of this, or any other, IMF-dependent model. Our model, which uses four input variables, $K p$ and IMF $\left(B_{x}, B_{y}, B_{z}\right)$, is not intended to provide physical insight into solar wind-magnetospheric processes but rather to fill a pressing need in the ionospheric and thermospheric modeling communities. This need is particularly pressing with the success of the Dynamics Explorer (DE) mission. As a result of this mission, extensive data sets of thermospheric and ionospheric parameters for well-defined IMF orientations have been acquired. These data sets, especially for northward IMF orientations, contain phenomena that need global models to unravel the physics. However, both ionospheric and thermospheric models require a global timedependent description of the magnetospheric electric field as a Copyright 1986 by the American Geophysical Union.

Paper number 5A8333.

0148-0227/86/005A-8333\$05.00 zeroth-order input. In the following, we shall briefly review the theoretical and observational literature on how the magnetospheric electric field depends upon the IMF.

The two-cell nature of the magnetospheric electric field at ionospheric altitudes was theoretically predicted by Axford and Hines [1961], Piddington [1962], and Dungey [1961]. Conceptually, the incorporation of the electric field into a magnetospheric-ionospheric system was made by Vasyliunas [1972]. Volland $[1973,1975,1978]$ made the first attempts at qualitatively modeling the electric field by considering ionospheric conductivities and currents. He also attempted to semiempirically introduce an IMF dependence. The relative simplicity of the basic Volland models have made them the major electric field models used by the global ionospheric and thermospheric modeling community [Roble et al., 1982; Schunk and Walker, 1973; Schunk and Raitt, 1980; Sojka and Schunk, 1985]. Topological considerations of how IMF antiparallel merging on the magnetopause occurs led Crooker [1979] to qualitatively describe how the magnetospheric field at ionospheric altitudes would depend upon the IMF $B_{y} / B_{z}$ orientation. These results were consistent with a variety of ionospheric observations (see below). Since this Crooker study, several other workers have extended the use of antiparallel merging considerations to describe the magnetospheric electric field in the ionosphere [Reiff and Burch, 1985; Chiu et al., 1985]. However, these model results are purely qualitative.

Lyons [1985] mapped the solar wind electric field into the ionosphere by a simple IMF-dipole magnetic field superposition model. By using only a dipole to represent the earth's field, this study again leads to only qualitative results. Lyons himself points out the unrealistic nature of the predicted ionospheric electric fields. For northward IMF orientations, Lyons [1985] does theoretically predict how the "four-cell" distribution can be created.

An alternative approach to modeling the magnetospheric electric field in the ionosphere is to solve an ionospheric current and conductivity problem [Kamide et al., 1981; Feldstein et al., 1984]. This method replaces the electric field problem with that of defining how the global current system depends upon the IMF and how the conductivity model is related to the current system. Feldstein et al. [1984] show how this procedure leads to 
electric field distributions which depend upon the IMF. This work is still at an early stage of development and only gives slightly more than qualitative trends.

To date, theoretical models of the magnetospheric electric field or even its origin are very primitive or expensive to run. The major theoretical models of the magnetospheric electric field are those of Spiro et al. [1981], Harel et al. [1981], and Yasuhara et al. [1983]. These models successfully couple various ionospheric and magnetospheric processes in order to simulate the electric field in the ionosphere and magnetosphere. Unfortunately, the models are limited to auroral and mid-latitude ionospheric regions. In addition, it is, at present, too expensive to run these models as an input function for a large-scale ionosphericthermospheric simulation. By themselves, these models do not yield a quantitative description of the electric field at ionospheric altitudes as a function of IMF (especially northward).

Satellite measurements of the high-latitude ionospheric electric field made large-scale inferences of the magnetospheric electric field possible [Cauffman and Gurnett, 1971; Heppner, $1972,1973]$. Even with these early results, a simple dependence upon the $K \boldsymbol{p}$ magnetic index, as well as a higher-order IMF dependence, was found. Indeed, to date, the work of Heppner [1972] is the only major statistical study of ionospheric electric field signatures observed by satellites. The dawn-dusk results of these initial Heppner studies were extended to two-dimensional electric field maps by Heppner [1977]. These studies favored southward IMF configurations because it was found that for northward IMF in winter the polar cap electric field was very irregular, showing no large-scale pattern.

Burke et al. [1979] found that for "strongly" northward IMF, a four-cell electric field pattern is observed. This feature is present for only a small percentage of the time; statistically no exact figure is available yet. Following this observation, several other workers have carried out data studies for selected IMF orientations or specific regions of the ionosphere. Heelis and Hanson [1980] looked at whether electric field reversals were shear or rotational, Heelis [1984] looked at the cusp electric field geometry as a function of the IMF, and Burch et al. [1985] used the DE data base to consider IMF $B_{y}$ dependencies. Based upon discrepancies between the observed electric field and the Volland simple analytic models, Heelis et al. [1982] put forward an analytic model which has several free parameters that enables the user to modify a Volland two-cell pattern.

With the advent of the incoherent backscatter radars, a ground-based method of observing the ionospheric electric field became possible. From both Chatanika in Alaska and Millstone Hill in Massachusetts, the electric field has been mapped as a function of local time up to invariant latitudes of about $72^{\circ}$ [Evans et al., 1980; Foster et al., 1981]. Data from both radar sites have been used to produce ionospheric electric field models [Evans et al., 1980; Foster, 1983]. Unfortunately, these data sets only cover latitudes equatorward of about $72^{\circ}$ invariant latitude, a region not particularly dependent on the IMF. The Sondrestrom incoherent scatter radar, which is located in the polar cap, is expected to yield very useful data on the IMF dependence of the ionospheric electric field [Clauer et al., 1984; de la Beaujardiere et al., 1985]. A wide variety of ionospheric electric field measurements for different IMF orientations has already been published, although statistics on the occurrence frequency and correlation of signatures is lacking.

We have constructed our model using both quantitative and qualitative data from the above references. Current understand- ing of how the IMF-dependent electric field is generated inadequate that only empirical modeling is feasible. In sectio these empirical inputs are discussed and give rise to the mod The model is then presented in an ionospheric convection sito tion where corotation is an important ingredient. This leads to description in section 4 of sunward ionospheric plasma tran port in the polar cap for northward IMF orientations. In sectio 5 we discuss the validity of the model and areas in which mo empirical results are required.

\section{DESCRIPTION OF THE IMF-DEPENDENT MODEL}

Convection exhibits a two-celled nature during periods southward IMF, while during some periods when the IMF northward, a four-celled pattern with sunward convection ove the polar cap is seen. In this section a simple mathematica model is described which has these general features. Many of the parameters which make up the model are assumed to $b$ dependent upon magnetic activity (via the $K p$ index) as well a the interplanetary magnetic field. The dependence of some these parameters on the IMF and $K p$ is purely conjectural, ye for others there exists empirical evidence for our selection. In section 3 we present these parameters and the empirical evidence which supports some of the selected values.

For convenience, the ionosphere is assumed to be divided $b$ the polar cap boundary into two main regions. The ionospher potential is defined on each of these regions, and then the different regions are connected with appropriate boundary con ditions. The functional form of the potential in the two regions has been chosen so as to give two-celled convection with antisunward flow over the polar cap and return flow equatorward of the polar cap boundary. An additional potential is added to the polar cap region whenever the $z$ component of the interplane. tary magnetic field turns northward. This additional potential causes the ionospheric flow to be diverted into a four-celler pattern. The functional forms chosen for the potentials are nor described.

\subsection{Potential for $B_{z}$ Southward}

In the region outside of the polar cap the electric field if assumed to vary linearly with colatitude $\theta$. Starting from the polar cap boundary $\left(\theta=\theta_{\mathrm{pc}}\right)$, the meridional electric field increases from zero to a maximum at $\theta_{\max }$ and then decreasesto zero again at the equatorward boundary $\theta_{\text {eq }}$. These regions are shown schematically in Figure 1, where regions I and II an poleward and equatorward of the boundary, $\theta=\theta_{\max }$, respec tively. The electric potentials in these two regions are given by

$$
\Phi_{\mathrm{I}}(\theta, \phi)=\Phi_{\mathrm{pc}}(\phi, K p)\left[1-\frac{\left(\theta-\theta_{\mathrm{pc}}\right)^{2}}{\left(\theta_{\max }-\theta_{\mathrm{pc}}\right)\left(\theta_{\mathrm{eq}}-\theta_{\mathrm{pc}}\right)}\right]
$$

and

$$
\Phi_{\mathrm{II}}(\theta, \phi)=\Phi_{\mathrm{pc}}(\phi, K p) \frac{\left(\theta-\theta_{\mathrm{eq}}\right)^{2}}{\left(\theta_{\mathrm{eq}}-\theta_{\max }\right)\left(\theta_{\mathrm{eq}}-\theta_{\mathrm{pc}}\right)}
$$

where $\phi$ is the azimuthal angle starting from midnight, as show in Figure 1, and $\Phi_{\mathrm{pc}}(\phi)$ is the potential along the perimeter of the polar cap. Equatorward of the boundary, $\theta=\theta_{\text {eq }}$, the potentialis zero.

In the region inside of the polar cap boundary we assumethe potential to be given by 


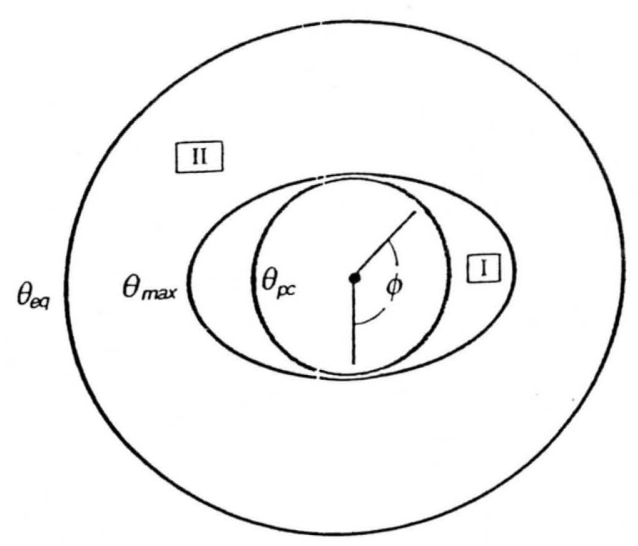

Fig. 1. A schematic representation of adopted convection boundaries in a polar coordinate system. The grey inner circle defines the polar cap region. The bottom of the figure is midnight with dawn on the right and the magnetic pole is at the center.

$$
\Phi(\theta, \phi)=\Phi_{\mathrm{pc}}\left(\phi=\phi_{x}, K p\right)
$$

$$
\cdot\left[\frac{\sin \theta \sin \phi}{\sqrt{\sin ^{2} \theta_{\mathrm{pc}}-\sin ^{2} \theta \cos ^{2} \phi}}-2 f(\mathbf{B}) \cos \left(\frac{\pi \theta}{2 \theta_{\mathrm{pc}}}\right)\right]
$$

where

$$
\phi_{x}=\sin ^{-1} \sqrt{1-\frac{\sin ^{2} \theta \cos ^{2} \phi}{\sin ^{2} \theta_{\mathrm{pc}}}}
$$

Note that the IMF-dependent function $f(\mathbf{B})$, which is defined in the section 3, forces an asymmetry between the flow in the morning and evening sectors within the polar cap.

\subsection{Additional Potential for $\boldsymbol{B}_{\mathbf{z}}$ Northward}

Whenever the $z$ component of the interplanetary magnetic field turns northward, an additional potential $\Phi^{\prime}$ is added to the polar cap region. The direction of the electric field due to this potential is such that it tends to oppose the dawn-dusk electric field which is normally present in the polar cap, and thus this additional potential acts to drive the plasma in a sunward direction. This additional or reverse potential is assumed to be

$$
\Phi^{\prime}(\theta, \phi)=-\Phi_{\mathrm{o}}^{\prime}(\mathbf{B})\left(\frac{d}{l}+f(\mathbf{B})\right)\left[1+\cos \left(\frac{\pi r^{\prime}}{r_{\mathrm{pc}}^{\prime}}\right)\right]
$$

within the polar cap $\left(\theta \leq \theta_{\mathrm{pc}}\right)$ and zero outside, where

$$
\begin{gathered}
d=\sin \theta \sin \phi+f(\mathbf{B}) \sin \theta_{\mathrm{pc}} \\
l=\sin \theta_{\mathrm{pc}}(1-|f(\mathbf{B})|)
\end{gathered}
$$

and

$$
\begin{gathered}
\left(\frac{r^{\prime}}{r_{\mathrm{pc}}^{\prime}}\right)^{-1}=\frac{\sin \theta_{\mathrm{pc}}}{d^{2}+\sin ^{2} \theta \cos ^{2} \phi} \\
\cdot\left[f(\mathbf{B}) d+\sqrt{d^{2}+\sin ^{2} \theta \cos ^{2} \phi\left(1-f^{2}(\mathbf{B})\right)}\right]
\end{gathered}
$$

Again, as in (2), the IMF-dependent function $f(\mathbf{B})$ creates an asymmetry in the polar cap flow.

\section{Empirically Suggested Relationships}

As noted above, our model contains parameters, such as the size of the polar cap region, $\theta_{\mathrm{pc}}$, which are presumed to depend on the $K p$ index and on the interplanetary magnetic field. Whenever possible, we have tried to model this dependence using empirical evidence. This modeling is described in this section.

\subsection{Southward IMF With $B_{y}=0$}

For a southward IMF the predominant signature of ionospheric convection is a two-cell pattern with antisunward flow over the polar cap. The magnitude of this flow is roughly proportional to the level of magnetic activity, as given by the $K p$ index. Indeed, the variation of the total potential drop across the polar cap as a function of $K p$ has been studied by various workers [Heppner, 1973; Kivelson, 1976; Reiff et al., 1981]. This variation is statistically the best known of the electric field dependencies. Heppner [1973] quotes a potential of $20 \mathrm{kV}$ for a $K p$ of 0 and $100 \mathrm{kV}$ for a $K p$ of 6 . A linear $K p$ relationship based upon these values is statistically consistent with the literature for this dependence. Thus we have adopted the relationship

$$
\Phi_{0}(K p)=10+6.5 K p
$$

where $\Phi_{0}$ is one half of the total potential drop across the polar cap in kilovolts. To date, this dependence appears to be valid for all orientations of the IMF.

The ionospheric electric field is broken into two regions: a polar cap region and an equatorward region. These two basic regions differ significantly in that the polar regions, both north and south, can respond quite independently to the IMF, while the equatorial regions, being on closed field lines, are strongly coupled between hemispheres. The boundary between the polar and equatorial regions is assumed to be a circle. Heppner [1972, 1973] presented restricted dawn-dusk satellite observations of the ionospheric electric field, which gives some justification for this assumption in the dawn-dusk plane. Indeed, in Heppner [1977] a $K p$ dependence of this radius is indicated; as $K p$ increases, the radius increases. Hence we adopted the relationship

$$
\theta_{\mathrm{pc}}=15+0.3 \mathrm{Kp}
$$

for the colatitude $\theta_{\mathrm{pc}}$ of the polar cap boundary (in degrees). This relationship is consistent with the model of Volland [1975].

The Chatanika and Millstone Hill radars only reach invariant latitudes of about $73^{\circ}$ and hence in the dawn-dusk sector cannot measure the polar cap velocity reversal except under extremely active conditions [Evans et al., 1980; Foster et al., 1981]. Thus (5) is consistent with the radar data as well.

In the noon-midnight plane the assumption that the polar cap boundary is a circle is less obvious. Around noon the "throat" [Spiro et al., 1978; Heelis, 1984] and around midnight the "Harang" features severely distort ionospheric convection. The Chatanika and Millstone Hill radars have observed the night sector Harang region to move well below $73^{\circ}$ invariant, while the noon cusp is almost always significantly poleward of $73^{\circ}$. Based upon the radar data and the Heelis [1984] cusp data, we have assumed that the polar cap circle has an antisunward offset from the magnetic pole. Heppner [1977] suggests that in the dawn-dusk plane the polar cap circle might also be offset toward either dawn or dusk. For simplicity we chose to center the reference frame of our potential model, used in section 2 , at $85^{\circ}$ magnetic latitude on the midnight meridian. Thus note that a 
transformation is necessary between geomagnetic coordinates and the coordinates used for the model. A consequence of which is that the colatitude used in section 2 cannot be readily converted to invariant latitude or $L$ value.

A variety of observations suggest that not only should the polar cap be offset in an antisunward direction but that it should also be rotated 1-2 hours toward morning [Spiro et al., 1978; Heelis, 1984; Yasuhara et al., 1983]. These findings are also consistent with both the Chatanika and Millstone Hill radar observations [Foster, 1983; Evans et al., 1980]. Theoretically, this rotation is believed to be due to an enhanced Hall conductivity in the noon sector auroral zone [Yasuhara et al. 1983]. In the present work this rotation is not included, but in ionospheric modeling, it could be included by an appropriate coordinate transformation before computing the potential.

In section 2 the specification of the electric field requires the potential to be defined on the polar cap/boundary. Volland [1975] selected this potential so that the electric field was constant within the polar cap. However, in the noon and midnight regions the electric field is relatively large and structured in comparison with the rest of the polar cap. The noon throat region is identified by enhanced electric fields [Spiro et al., 1978, 1981; Heelis, 1984], while in the midnight Harang region, discrete auroral forms are directly correlated with electric field structures [Gurnett and Frank, 1973]. Unfortunately, neither region is understood to the extent that detailed modeling is realistic. To overcome this problem, Heelis et al. [1982] used a simple procedure of defining the potential around the polar cap circle such that the high and low electric fields occurred in the observed regions. We followed this procedure and assumed the potential on the polar cap boundary to be

$$
\begin{array}{lrl}
\Phi_{\mathrm{pc}}(\phi, K p) & =\Phi_{0}(K p) \sin (3 \phi / 2) & -\pi / 3<\phi<\pi / 3 \\
\Phi_{\mathrm{pc}}(\phi, K p) & =\Phi_{0}(K p) & \pi / 3 \leq \phi \leq 2 \pi / 3 \\
\Phi_{\mathrm{pc}}(\phi, K p) & =-\Phi_{0}(K p) \cos (3 \phi / 2) & 2 \pi / 3 \leq \phi<4 \pi / 3 \\
\Phi_{\mathrm{pc}}(\phi, K p) & =-\Phi_{0}(K p) & 4 \pi / 3 \leq \phi \leq 5 \pi / 3
\end{array}
$$

Volland [1975] modeled the falloff of the potential equatorward of the polar cap with a function that varied with the inverse sine of the colatitude, the exponent of this function being determined by observation [cf. Sojka et al., 1980]. Heppner [1977] pointed out that this function was not consistent with observations in at least two major ways. First, the Volland function placed the maximum electric field at the polar cap edge, leading to a strong velocity shear at this edge. Second, the data seemed more consistent with the electric field being zero below some colatitude $\theta_{\text {eq. }}$. From Heppner [1977] this minimum latitude can be described as a circle which expands equatorward with increasing $K p$, except in the noon sector where $\theta_{\text {eq }}$ is independent of $K p$. At noon the location of the boundary where the potential becomes zero was found to be near $70^{\circ}$ invariant latitude. This result is consistent with both radar and satellite observations [Evans et al., 1980; Foster et al., 1981; Spiro et al., 1978; Heelis, 1984]. We have modeled this dependence by

$$
\begin{array}{lrl}
\theta_{\text {eq }}(\phi, K p) & =25+2 K p & -\pi / 2 \leq \phi \leq \pi / 2 \\
\theta_{\text {eq }}(\phi, K p) & =25+2 K p(1+\cos \phi) & \pi / 2<\phi<3 \pi / 2
\end{array}
$$

where the polar cap offset of $5^{\circ}$ has been taken into account so as to place this boundary at $70^{\circ}$ invariant latitude at $\phi=\pi$. If an offset other than $5^{\circ}$ is being used, then the equation should also be changed.
Between $\theta_{\mathrm{pc}}$ and $\phi_{\mathrm{eq}}$, Heppner [1977] found that the electric field reached a maximum value at some location $\phi_{\max }$. Along the dawn and dusk meridians, a boundary where the electric field reaches a maximum is suggested by the other data as well [Heelis and Hanson, 1980]. However (in either the noon or the midnight sectors), there is no strong evidence for an equatorward offset of $\theta_{\max }$. Thus we have adopted

$$
\theta_{\max }(\phi, K p)=\theta_{\mathrm{pc}}(K p)+0.3\left[\theta_{\mathrm{eq}}(\phi, K p)-\theta_{\mathrm{pc}}(K p)\right]|\sin \phi|(8)
$$

for the boundary at which the electric field reaches a maximum value. Although Heppner [1977] suggested that the location of this maximum may be seasonally dependent, we have not included this dependence.

Having adopted the above relationships, the ionospheric electric field can be obtained from equations (1) and (2) (assum. ing the IMF dependence to be given by $f(\mathbf{B})=0$ for now). Figure $2 a$ shows contours of the equipotentials for this model for a $K p$ $=2.5$ and $B_{z}$ southward. The contours are drawn in the MLT. magnetic latitude coordinate system and are labeled in kilovolts. In the dusk sector the contours are dashed, indicating a negative potential. The polar cap electric fields are largest near the noon and midnight polar cap boundaries. Higher electric fields can be obtained by increasing the extent of the regions of constant potential in the dawn and dusk sectors in equation (6).

Figures $2 b$ and $2 c$ show the dawn-dusk potential and electric field distributions, respectively. Both the potential and electric field are plotted as a function of magnetic latitude going from dusk over the pole to dawn. The polar cap region shows a uniform electric field of $14 \mathrm{mV} / \mathrm{m}$, the calculation being done for an altitude of $300 \mathrm{~km}$. Outside the polar cap, the electric field has a peak value of $32 \mathrm{mV} / \mathrm{m}$ at a latitude just equatorward of the polar cap. The general shape of the dawn-dusk potential and electric field are similar to the features in the Heppner [1977] and Volland [1975] models, but at lower latitudes they are dissimilar to the features in the Volland [1975] model.

\subsection{Southward IMF With $B_{y} \neq 0$}

The electric field has been found to significantly correlate with $B_{y}$ only in the polar cap. Heppner $[1972,1973]$ showed how the dawn-dusk electric field depends on $B_{y}$, while Heelis [1984] showed how the throat region also changed with $B_{y}$. Other observations have found the same dependence on $B_{y}$ [Potemra et al., 1984; Clauer et al., 1984; de la Beaujardiere et al., 1985; Burch et al., 1985]. These observations are described in a qualitative manner by the antiparallel merging model [Crooker, 1979]. For positive $B_{y}$ in the northern hemisphere the electric field is enhanced in the region of the dawn polar cap, while in the southern hemisphere the dusk electric field is enhanced. For negative $B_{y}$ the simple Crooker model gives the opposite electric field variation. Observationally, this symmetry in $B_{y}$ is not certain, since Heppner [1977] did not see a strong dusk electric field for the negative $B_{y}$ case. Theoretically, this may arise from a noon-midnight gradient in the ionospheric conductivity which tends to enhance the polar cap electric field in the dawn sector [Atkinson and Hutchinson, 1978], although this asymmetry in not included in our model.

Heelis [1984] suggests that the $B_{y}$ dependence may be more of a $B_{x}, B_{y}$ dependence in that an IMF coordinate system taking into account the "hose pipe" angle could be more important. This suggestion is also supported by the early Sondrestrom data [de la Beaujardiere et al., 1985], although the Clauer et al. [1984] 

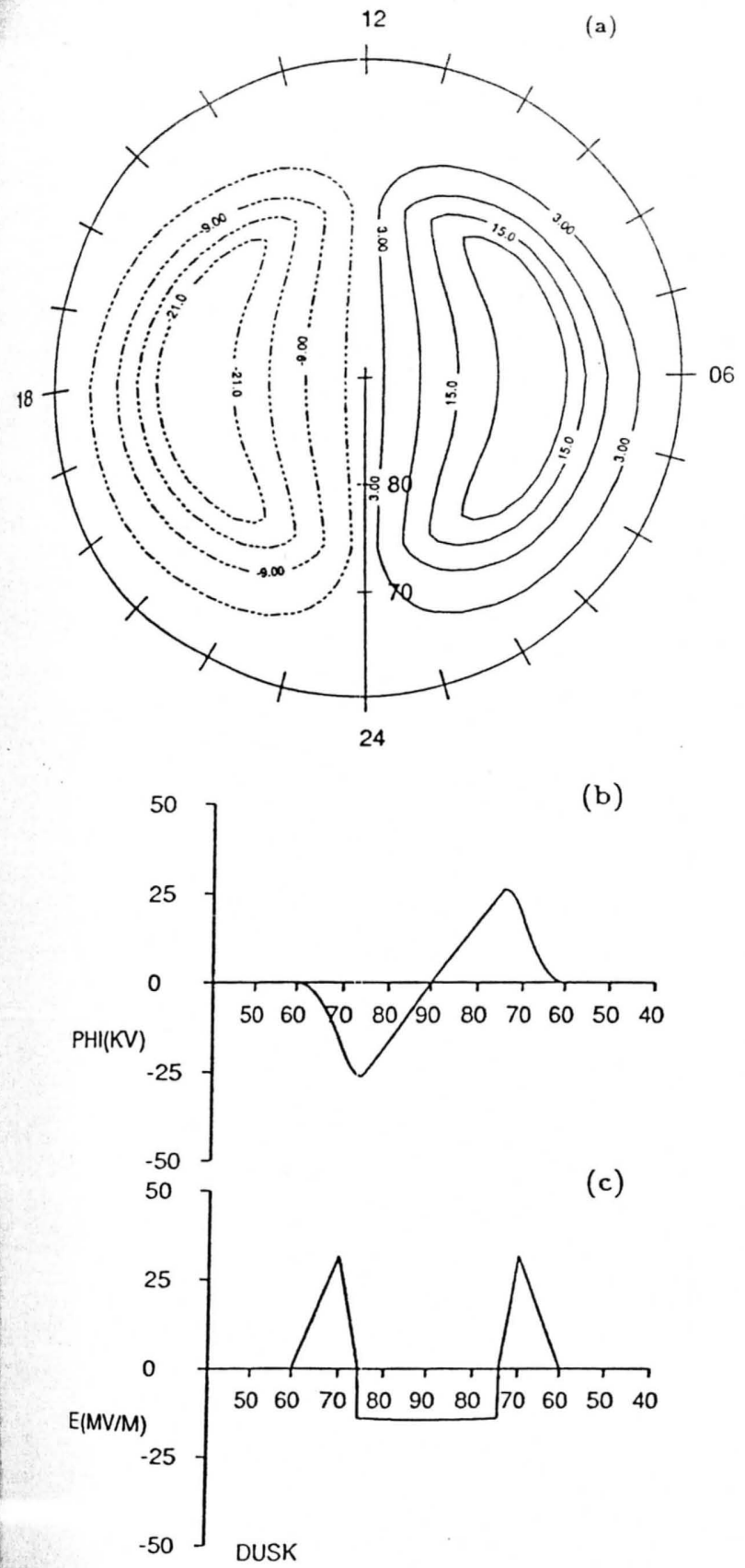

Fig. 2. Model of the northern hemisphere magnetospheric field for a southward IMF orientation $\left(B_{x}=0, B_{y}=0, B_{z}=-6 \gamma\right)$. (a) Contours of electric potential in an MLT-magnetic latitude frame. The potential contours are at 6-kV intervals. (b) The electric potential along the dusk to dawn meridian. $(c)$ The electric field along this meridian.

data do not support this viewpoint. We have not included a $B_{x}$ dependence, although if such a dependence is found to be mportant, then the $B_{x}, B_{y}$ coordinate system could be rotated to a plane where a single parameter would describe the depen-
dence. The $B_{y}$ dependence noted above is modeled by the function
$f(B)$ in equations (2) and (3). We have adopted the relationship

$$
f(\mathbf{B})=\frac{0.35 B_{y}}{\sqrt{B_{y}^{2}+B_{z}^{2}}}
$$

Figure $3 a$ shows contours of constant potential in the northern hemisphere for $K p=2.5, B_{y}=6 \gamma$, and $B_{z}=-6 \gamma$. The coordinate system is the same as that of Figure $2 a$, and the same contour levels have been drawn. A comparison of Figures $2 a$ and $3 a$ indicates that the enhanced electric field in the dawn sector is prominent for $\boldsymbol{B}_{\boldsymbol{y}}>\mathbf{0}$, as is the skewing of the two convection cells. The dusk cell has expanded in size and occupies about half of the dawn sector polar cap. The throat feature has asymmetries about noon which are like those of Heelis [1984]. Because of the symmetry used to construct the $B_{y}$ dependence, the negative $B_{y}$ case would give the same pattern reflected about the noon-midnight meridian.

Figures $3 b$ and $3 c$ show the dawn-dusk potential and electric field distribution, again for the same coordinates as used in Figure 2. Equatorward of the polar cap the distributions are the same as in Figures $2 b$ and $2 c$; however, in the polar cap they differ. The dusk electric field is reduced from 14 to $3 \mathrm{mV} / \mathrm{m}$, while the dawn electric field is increased to $25 \mathrm{mV} / \mathrm{m}$.

\subsection{Northward IMF}

Since 1979, when Burke et al. [1979] published data suggesting four-cell convection with sunward flow in the polar cap for a northward IMF, various other studies have been carried out to define the northward convection pattern [Potemra et al., 1984; Lyons, 1985; Reiff and Burch, 1985; Chiu et al., 1985]. Recent interest has also been generated by the extensive DE observations of well-defined Theta-auroral signatures for northward IMF.

We have modeled the noted sunward convection by introducing a small region of reverse potential within the polar cap. The magnitude of this reverse potential has been roughly estimated from the electric field data of Burke et al. [1979] to be

$$
\Phi_{0}^{\prime}(\mathbf{B})=\frac{\left(9 B_{z}+30\right)}{4} \sqrt{\frac{B_{z}^{2}}{B_{y}^{2}+B_{z}^{2}}}
$$

For the case of purely northward IMF this region of reverse potential is centered on the noon-midnight meridian. However, for nonzero $B_{y}$ this pattern is seen to lie in the region of the larger of the two original cells [Reiff and Burch, 1985; Chiu et al., 1985].

Because electric fields from the reverse potential are added to relatively strong dawn-dusk fields, the northward component of the IMF must be above some threshold value before four-cell convection is seen. For the value of the reverse potential given in (3) this threshold is about $1 \gamma$ during very quite conditions. As magnetic activity increases, the dawn-dusk fields increase even more (see equation (4)), and $\boldsymbol{B}_{z}$ must be more strongly northward before four-cell convection is created.

After considering several schematical representations of ionospheric convection obtained from studies during periods of northward IMF [Potemra et al., 1984; Reiff and Burch, 1985; Chiu et al., 1985], we concluded that the extreme $B_{y}$ cases which appear as three-cell patterns can be described as four-cell patterns with one cell becoming very small. In particular, the study of Potemra et al. [1984] indicates that it is necessary for a fourth cell to exist, even if small, for consistency with their field-aligned current observations.

Figure $4 a$ shows ionospheric convection for a purely northward IMF where $B_{z}=6 \gamma$ and $K p=1$. The contours are in the same polar coordinate system as for Figure 2. A comparison of these contours with those of Figure $2 a$ shows qualitatively how 

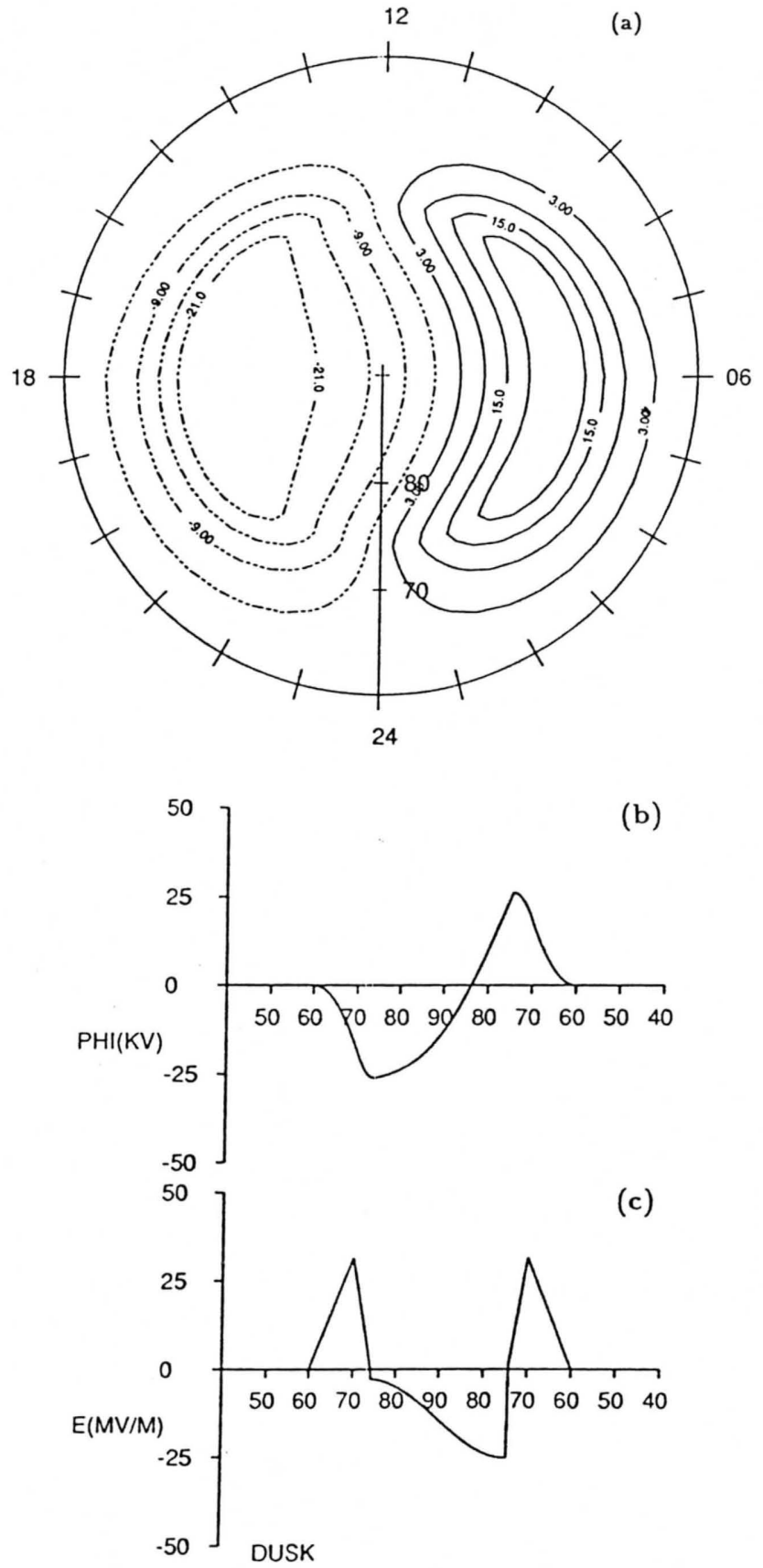

Fig. 3. Identical to Figure 2 except that the southward IMF orientation is $B_{x}=0, B_{y}=6 \gamma, B_{z}=-6 \gamma$. The potential contours are at $6-\mathrm{kV}$ intervals.

we have included the two reverse convection cells inside the polar cap region. For this example, the potential across these two inner cells is $11 \mathrm{kV}$ with the electric field in the dawn-todusk direction opposing that of the original polar cap field. A major portion of the original two cells remains unchanged. In addition, the general pattern for this IMF orientation is similar to the published schematics noted above.

The dawn-dusk potential and electric field are shown in Figures $4 b$ and $4 c$, respectively. Figure $4 c$ shows the region of sunward plasma convection as a region in the center of the polar cap with a positive electric field. The peak electric field in the sunward convection region reaches $15 \mathrm{mV} / \mathrm{m}$. This magnitude is similar to those observed by Burke et al. [1979]. For this IMF orientation the sunward flow region is along the midnight $-n_{00}$ meridian and restricted to a circle of about $5^{\circ}$ centered at the middle of the polar cap circle.

The electric potential distribution, the dawn-dusk potential and the dawn-dusk electric field are shown in Figure 5 for the case of a northward IMF with $B_{y}=6 \gamma$. Figure 5 is in the same format as Figure 4. The plot is for the northern hemisphere with $K p=1$ and $B_{z}=6 \gamma$. In this example the dawn electric field in the polar cap is enhanced in a manner similar to the corresponding southward case (see Figure 3). The dusk cell extends into the dawn sector and superimposed upon it are two additional cells. One of these additional cells is centered about the pole and
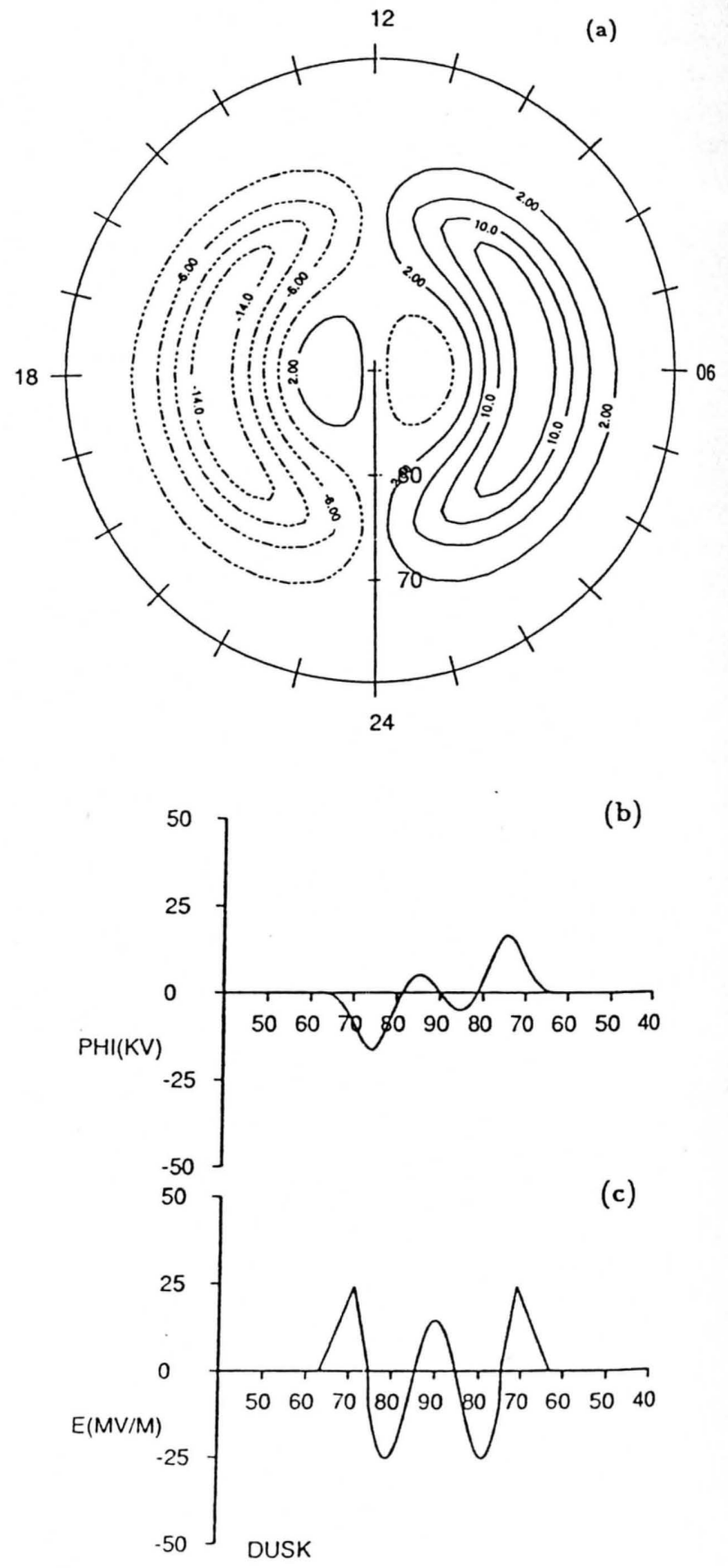

Fig. 4. Identical to Figure 2 except that the IMF orientation is northward with $B_{x}=0, B_{y}=0, B_{z}=6 \gamma$. The potential contours are at $4-\mathrm{kV}$ intervals. 


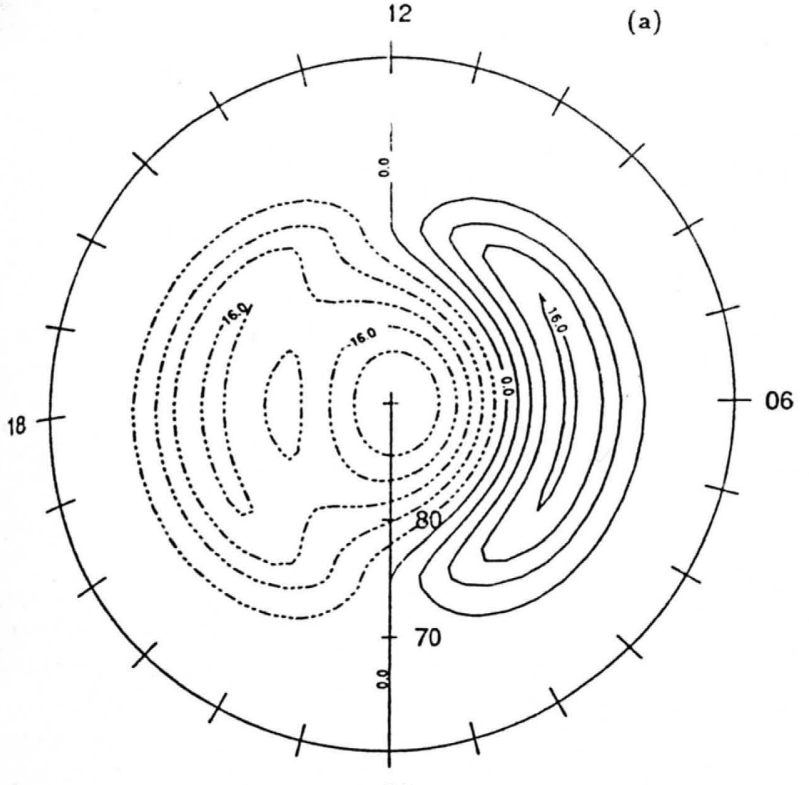

24

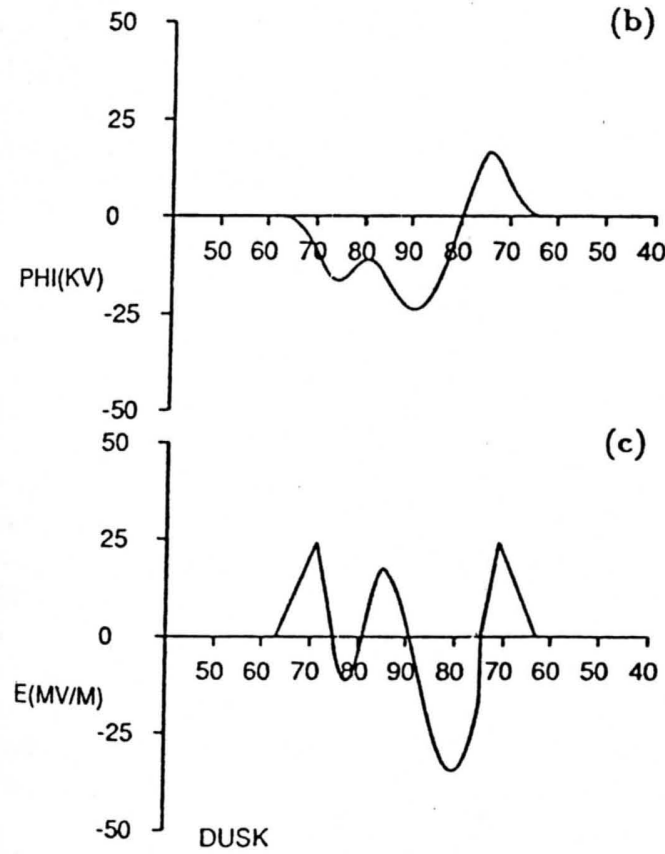

Fig. 5. Identical to Figure 2 except that the IMF orientation is northward with $B_{x}=0, B_{y}=6 \gamma, B_{z}=6 \gamma$. The potential contours are at 4-kV intervals.

corresponds to clockwise convection. The smaller cell is located at $1800 \mathrm{MLT}\left(80^{\circ}\right.$ latitude) and corresponds to counterclockwise convection. What appears as a third closed cell at 1800 MLT $\left(45^{\circ}\right.$ latitude) is considered to be part of the original dusk cell. For even stronger $B_{y}$ relative to $B_{z}$ orientations the counterclockwise cell effectively disappears.

The pattern shown in Figure $5 a$ is similar to the schematics referred to earlier. Unfortunately, there are almost no published data with which Figures $5 b$ and $5 c$ can be compared. In Figures $5 b$ and $5 c$ the dawn-dusk potential and electric field are shown. The region of sunward convection has moved towards dusk (see electric $5 c$ ). In the region of sunward convection the maximum convectield is $17 \mathrm{mV} / \mathrm{m}$, and the potential across this reverse convection region is $6 \mathrm{kV}$.

\section{IONOSPHERIC Plasma CONVECTION}

Having developed an IMF convection model, it is now possible to quantitatively look at how this will impact the ionosphere. In the high-latitude $F$ region, plasma transport is dominated by the magnetospheric electric field. However, the corotation electric field, although small, does have a noticeable effect. Combining these two electric fields enables the $F$ region plasma $\mathbf{E} \times \mathbf{B}$ velocity to be calculated. Once this velocity is available, the plasma trajectory and circulation period can be calculated. These parameters can then be used to determine in which regions of the ionosphere high or low densities can be
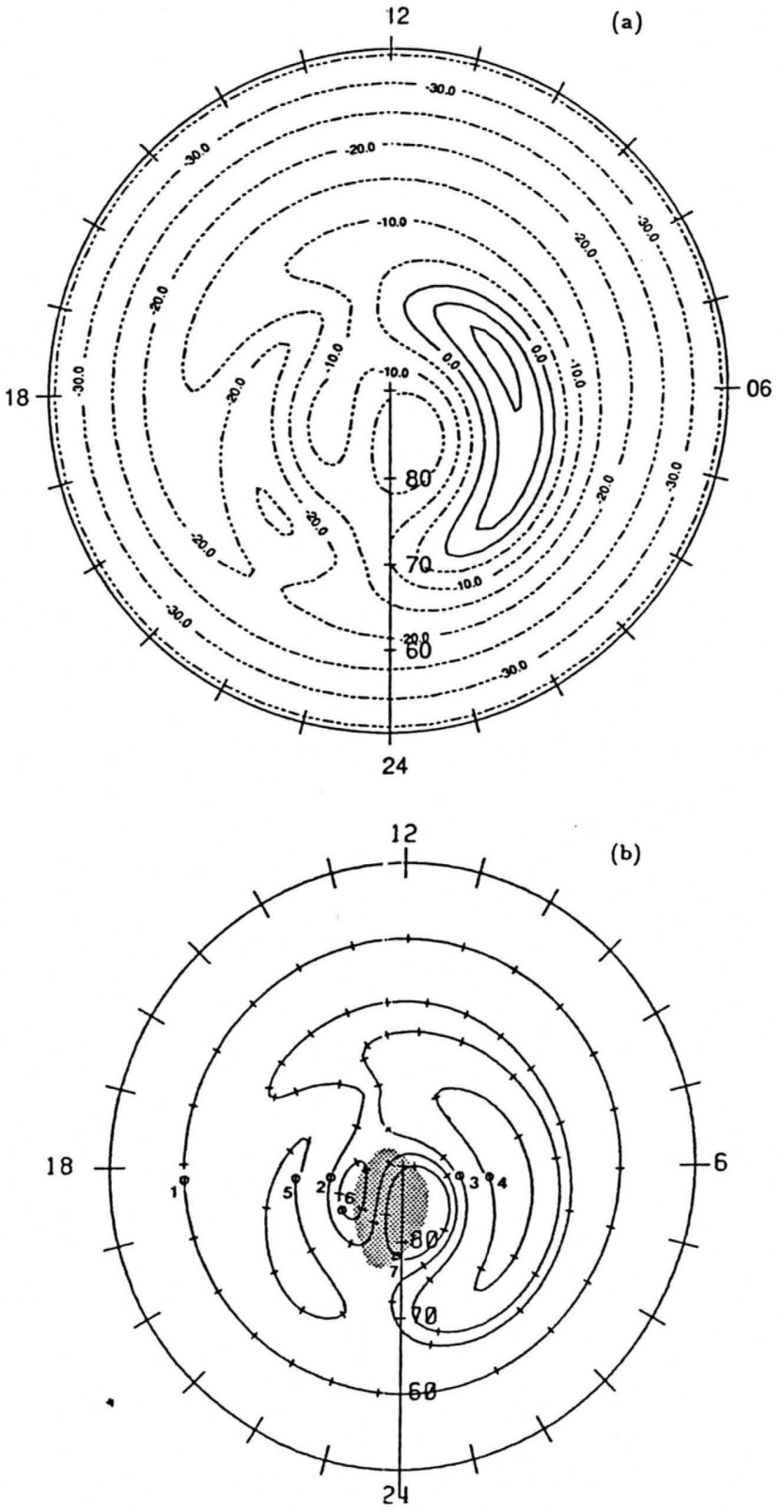

Fig. 6. (a) A model of the northern hemisphere magnetospheric potential plus corotational potential contours for a northward IMF orientation of $B_{x}=0, B_{y}=3 \gamma, B_{z}=6 \gamma$. The contours are drawn at $5-\mathrm{kV}$ intervals and presented in an MLT-magnetic latitude frame. (b) A selection of ionospheric plasma convection trajectories for this IMF; each trajectory has tick marks at hourly convection intervals around the trajectory. The shading shows the region of sunward convection in the polar cap. The start of each trajectory is marked with a circle. 

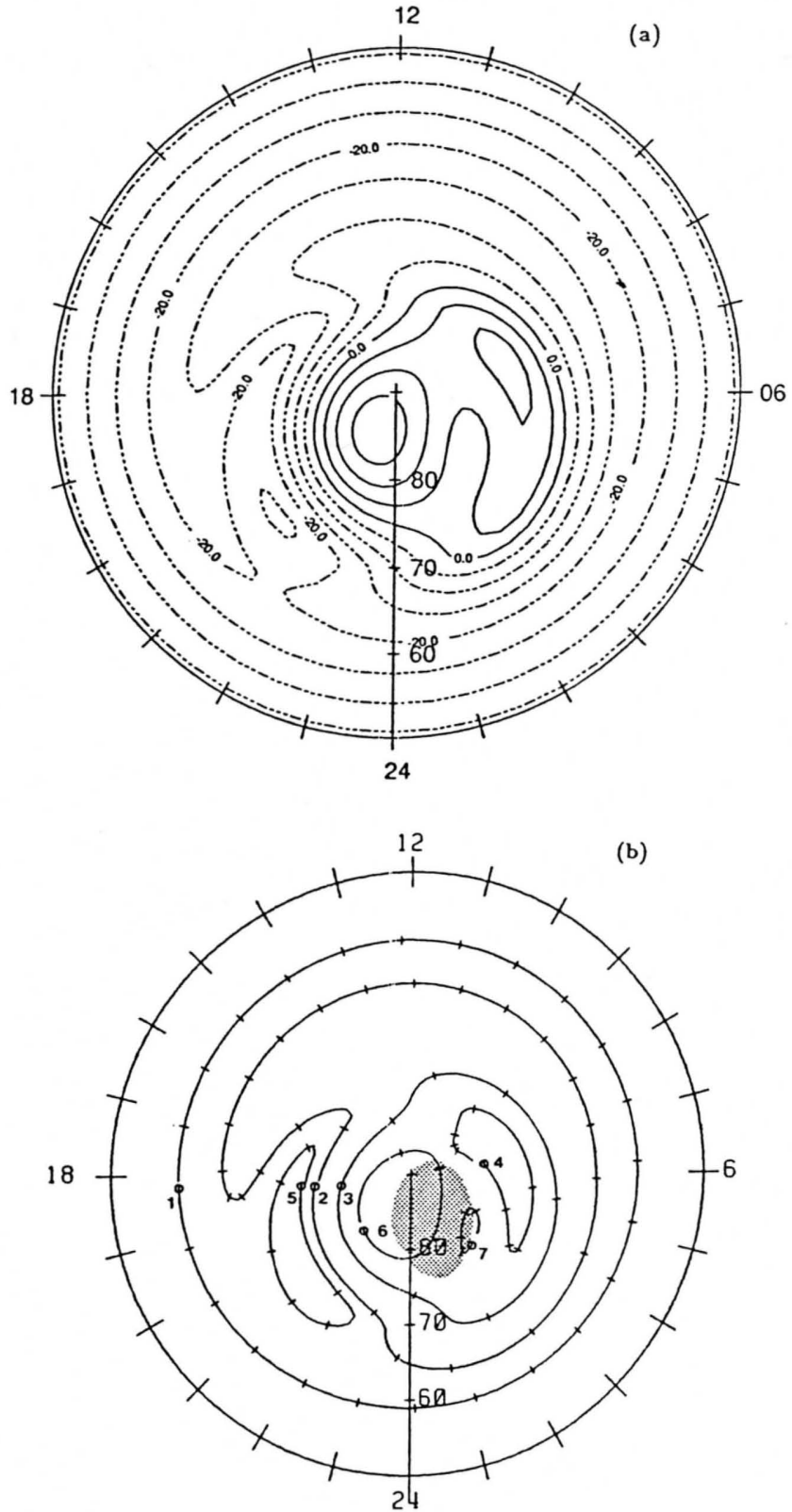

Fig. 7. Identical to Figure 6 except that the northward IMF orientation is changed to $B_{x}=0, B_{y}=-3 \gamma, B_{z}=6 \gamma$.

expected due to combinations of high- or low-speed flow with, or without, plasma production. In our earlier high-latitude ionospheric studies we showed how these parameters were important indicators of ionospheric morphology for simple two-cell plasma convection patterns [Sojka et al., 1979, 1981; Sojka and Schunk, 1983]. The main transport concern in these studies was how fast the antisunward flow over the polar cap was or where in the dusk sector did plasma stagnation give rise to the mid-latitude trough. With the existence of sunward flow in the polar cap for strongly northward IMF orientations, an entirely new aspect of plasma transport can be studied.

Figure $6 a$ shows contours of equipotential for the combined corotation and magnetospheric electric fields for an IMF orientation of $B_{x}=0 \gamma, B_{y}=3 \gamma, B_{z}=6 \gamma$, and $K p=1$. The equipotentials are drawn at $5-\mathrm{kV}$ intervals, and the nonzero potentials equatorward of the polar region indicate the dominance of corotation. This plot is for the northern hemisphere. These equipotentials represent plasma convection trajectories; at lon latitudes the circles represent corotation, while at high latituden complex trajectories arise from the four-cell nature of the map netospheric field. On the dawnside of the polar cap the equi potentials are all bunched together, indicating relatively fast antisunward convection. However, on the duskside, two small convection regions separated by an "s"-shaped trajectory are present. Figure $6 b$ shows seven individual plasma trajectorie plotted in a similar coordinate system for the same IMF condi. tions. Each trajectory is identified by a number, and tick marks are drawn at 1 -hour intervals along the trajectory. Trajectories? and 3 trace similar paths at low latitudes but cross the polarcap in quite different ways. Plasma following trajectory 2 stagnates near $1800 \mathrm{MLT}$ at $68^{\circ}$ and then meanders between all four convection cells. In contrast, plasma following trajectory 3 cuts across the dawn polar cap, circulating around only the outer dawn cell. A region of sunward convection exists in the dusk sector of the polar cap between trajectories 6 and 7 . The shading has been added to highlight the extent of this region.

Figures $7 a$ and $7 b$ correspond to Figures $6 a$ and $6 b$, respec. tively, for an IMF orientation of $B_{x}=0 \gamma, B_{y}=-3 \gamma, B_{z}=6 \gamma$, and $K p=1$. Figure 7 is also for the northern hemisphere. The most striking difference between Figures $6 a$ and $7 a$ is that they are not mirror images of each other. Our IMF $B_{y}$ dependence is such that positive and negative $B_{y}$ effects are mirror images of each other. However, when corotation is included in the iono. sphere, this symmetry is removed. A consequence of this is that the ionosphere's response to positive and negative $B_{y}$ convection effects will be different. In Figure $7 b$ a set of seven trajectoriesis shown to highlight the different convection regions. Trajectory 3 encompassed three cells, the dawn cell and the two polar cap cells. Unlike trajectory 2 in Figure $6 b$, for this case (Figure $7 b$ ) no plasma trajectory meanders between all the cells. As a result of the two polar cap sections of trajectories ( 2 and 3 ) being confined in a small region in Figure $7 b$, the plasma densitiesin this region will have undergone similar histories. In contrast, in Figure $6 b$ the two polar cap sections of trajectories ( 2 and 3 ) are separated by a region containing a plasma convection cell; hence they have a completely different history and densities.

Another way to view these differences in past history is to look at the plasma circulation time around the trajectories. Tables 1 and 2 give these times for the trajectories in Figures $6 b$ and $7 b$, respectively. In Table 2 the three trajectories 3,6 , and?, which define the extent and "history" of the sunward flow region, have circulation periods between $31 / 2$ and $61 / 2$ hours. The corresponding sunward convection region in Figure $6 b$, trajer tories 2,6 , and 7 , have circulation periods between $3 \frac{1}{2}$ and 24 hours (see Table 1). These contrasting time scales are also rele vant when the dynamics of the magnetosphere is taken into consideration. The times listed in Tables 1 and 2 indicate the length of time the magnetospheric electric field pattern must remain constant for a single plasma flux tube to circulate onct

TABLE 1. Plasma Circulation Periods Associated With Figure 60

\begin{tabular}{cc}
\hline Trajectory & Circulation Period, hours \\
\hline 1 & 24.1 \\
2 & 23.8 \\
3 & 13.2 \\
4 & 4.9 \\
5 & 7.6 \\
6 & 4.1 \\
7 & 3.8 \\
\hline
\end{tabular}


TABLE 2. Plasma Circulation Periods Associated With Figure $7 b$ Trajectory Circulation Period, hours

\begin{tabular}{lr}
1 & 24.0 \\
2 & 23.4 \\
3 & 6.6 \\
4 & 8.9 \\
5 & 7.0 \\
6 & 3.8 \\
7 & 5.9 \\
\hline
\end{tabular}

around the ionosphere. The magnetosphere may remain constant for time scales of the order of 1-3 hours; however, time scales greater than 10 hours are uncommon. The ramifications of these considerations are discussed in section 5 .

\section{SUMMARY}

We have developed an IMF-dependent model of the magnetospheric electric field at ionospheric altitudes based upon published observations, qualitative models, and a limited understanding of the electric field source. The model has the following specific dependencies:

1. The $K p$ index controls the cross-tail potential, the diameter of the polar cap, and the extent of the equatorial falloff region.

2. For southward IMF orientations the model depends only on the IMF $B_{y}$ component and its relative orientation in the $\boldsymbol{B}_{y}$, $B_{z}$ plane. There is no $B_{x}$ dependence.

3. A simple $B_{x}$ dependence could be introduced by a rotational transformation of the IMF about $B_{z}$ such that a new $B_{y}$ direction could be defined as a combination of the original $B_{y}$, $B_{x}$ components.

4. The $B_{y}$ dependence, namely, both weak and strong electric field sectors in the polar cap, has opposite effects in the two hemispheres.

5. For northward IMF orientations a pair of extra convection cells exist inside the polar cap, which give rise to a region of sunward convection. The location of this sunward convection region moves into the dawn or dusk sector depending upon $B_{y}$ and the hemisphere.

The basic model contains a high degree of symmetry in the electric field dependence upon the polarity of $B_{y}$. When the corotation field is introduced, the resulting ionospheric patterns no longer show this symmetry. In the polar cap regions under northward IMF conditions the existence of a corotational field has not yet been proven. The question of its existence should be pursued both theoretically and experimentally since its presence would have an effect on ionospheric convection even in the polar cap.

Our model has been developed for constant IMF orientations. The validity of our assumption that a fixed IMF orientation produces a fixed ionospheric convection pattern is, at the present time, difficult to verify. After a change in the IMF orientation there would be a time delay before the ionospheric convection pattern changed. This time period may be as short as a few minutes, the Alfvén propagation speed from the magnetopause to the ionosphere, or significantly longer if horizontal propagation due to flux tube transport across the polar cap is prortant in setting up the new convection pattern. The latter process may lead to time constants of several hours. Complexities associated with the inertia of the atmosphere may lead to thermospheric-ionospheric generated electric fields during the one from one ionospheric convection pattern to a new Again, a time period of several hours may be involved.
The ramifications of this time "delay" in the ionosphere's electric field response can best be seen from data. Heppner [1973] published two sets of examples of dawn-dusk electric field crossings for selected IMF periods. His first example, Heppner [1973, Figure 8], is an orbit taken $\sim 4$ hours into a northward IMF period, showing a well-defined sunward convection region in the middle of the polar cap. The subsequent orbit shows a similar sunward convection region, although the IMF had very definitely turned southward just prior to the second orbit. A dawn-dusk orbit crosses flux tubes in the polar cap which were not directly connected to the dayside magnetopause, and hence no direct correspondence between these data and the current IMF would necessarily be expected, but rather a correlation with IMF a few hours earlier. Heppner [1973, Figure 9] also shows a set of five successive orbits during a period when the IMF reverses several times on a one to 2-hour time scale. The data all show very similar electric field patterns. Again, the data do not show a "strong" response to the marked IMF northwardsouthward changes during this period.

In summary, it appears that the time period for the ionospheric convection pattern to respond to changes in the IMF orientation is longer than a few minutes; however, exactly how long is unknown. Hence our model could not be used for studies where the IMF fluctuates faster than about an hour between northward and southward orientations. Alternatively, the model would be reasonable if the IMF were relatively constant for periods greater than several hours. Although infrequent, such periods are found, and the DE mission has acquired significant data sets for such conditions. We have in fact developed this convection model so that we can study these periods with our global ionospheric model.

From our comparison of current studies of the convection electric field, it seems that a number of empirical studies still need to be made. First, there is a need for a large-scale statistical analysis of convection data for IMF conditions which have been constant for several hours. This study would, for the first time, give insight into the true IMF control of the ionospheric convection pattern. To date, the IMF-dependent convection studies have considered limited data events or restricted location. Second, coordinated multiple satellite and ground-based convection observations need to be used in an attempt to elucidate the convection electric field time constant. From the above discussion, this time constant could vary significantly from a few minutes to many hours depending upon the as yet unknown magnetosphere-solar wind interaction which results in the convection electric field. Finally, given that these selected data sets for "fixed" IMF orientations exist, it will be possible to study other effects, such as seasonal effects, a $\boldsymbol{B}_{\boldsymbol{y}} / \boldsymbol{B}_{x}$ dependence, a $\boldsymbol{B}_{\boldsymbol{z}}$ threshold for four-cell formation, and a nonsymmetric dependence upon $B_{y}$.

Acknowledgments. This research was supported by NASA grants NAG-5-455 and NAGW-77 to Utah State University. We thank one of the referees for the useful comments which simplified the polar cap potential description.

The Editor thanks two referees for their assistance in evaluating this paper.

\section{REFERENCES}

Atkinson, G., and D. Hutchinson, Effect of the day-night ionospheric conductivity gradient on polar cap convective flow, J. Geophys. Res., $83,725-729,1978$.

Axford, W. I., and C. O. Hines, A unifying theory of high-latitude geophysical phenomena and geomagnetic storms, Can. J. Phys., 39, 1433-1463, 1961. 
Burch, J. L., P. H. Reiff, J. D. Menietti, R. A. Heelis, W. B. Hanson, S. D. Shawhan, E. G. Shelley, M. Sugiura, D. R. Weimer, and J. D. Winningham, IMF $\boldsymbol{B}_{y}$-dependent plasma flow and Birkeland currents in the dayside magnetosphere, 1, Dynamics Explorer observations, J. Geophys. Res., 90, 1577-1593, 1985.

Burke, W. J., M. C. Kelley, R. C. Sagalyn, M. Smiddy, and S. T. Lai, Polar cap electric field structures with a northward interplanetary field, Geophys. Res. Lett., 6, 21-24, 1979.

Cauffman, D. P., and D. A. Gurnett, Double-probe measurements of convection electric fields with the Injun 5 satellite, J. Geophys. Res., 76, 6014-6027, 1971.

Chiu, Y. T., N. U. Crooker, and D. J. Gorney, Model of oval and polar cap arc configurations, J. Geophys. Res., 90, 5153-5157, 1985.

Clauer, C. R., P. M. Banks, A. Q. Smith, T. S. Jorgensen, E. FriisChristensen, S. Vennerstrom, V. B. Wickwar, J. D. Kelly, and J. Doupnik, Observation of interplanetary magnetic field and of ionospheric plasma convection in the vicinity of the dayside polar cleft, Geophys. Res. Lett., 11, 891-894, 1984.

Crooker, N. U., Dayside merging and cusp geometry, J. Geophys. Res., 84, 951-959, 1979.

de la Beaujardiere, O., V. B. Wickwar, J. D. Kelly, and J. H. King, Effect of the interplanetary magnetic field $y$ component on the high-latitude nightside convection, Geophys. Res. Lett., 12, 461-464, 1985.

Dungey, J. W., Interplanetary magnetic field and the auroral zones, Phys. Rev. Lett., 6, 47-48, 1961.

Evans, J. V., J. M. Holt, W. L. Oliver, and R. H. Wand, Millstone Hill incoherent scatter observations of auroral convection over $60^{\circ} \leq \lambda$ $\leq 75^{\circ}, 2$, Initial results, J. Geophys. Res., 85, 41-54, 1980.

Feldstein, Y. I., A. E. Levitin, D. S. Faermark, R. G. Afonina, and B. A. Belov, Electric fields and potential patterns in the high-latitude ionosphere for different situations in interplanetary space, Planet. Space Sci., 32, 907-923, 1984.

Foster, J. C., An empirical electric field model derived from Chatanika radar data, J. Geophys. Res., 88, 981-987, 1983.

Foster, J. C., J. R. Doupnik, and G. S. Stiles, Large-scale patterns of auroral ionospheric convection observed with the Chatanika radar, J. Geophys. Res., 86, 11,357-11,371, 1981.

Gurnett, D. A., and L. Frank, Observed relationships between electric fields and auroral particle precipitation, J. Geophys. Res., 78, 145-170, 1973.

Harel, M., R. A. Wolf, P. H. Reiff, R. W. Spiro, W. J. Burke, F. J. Rich, and M. Smiddy, Quantitative simulation of a magnetospheric substorm, 1, Model logic and overview, J. Geophys. Res., 86, 2217-2241, 1981.

Heelis, R. A., The effects of interplanetary magnetic field orientation on dayside high-latitude ionospheric convection, J. Geophys. Res., 89, 2873-2880, 1984.

Heelis, R. A., and W. B. Hanson, High-latitude ion convection in the nighttime $F$ region, J. Geophys. Res., 85, 1995-2002, 1980.

Heelis, R. A., J. K. Lowell, and R. W. Spiro, A model of the highlatitude ionospheric convection pattern, J. Geophys. Res., 87, 6339-6345, 1982.

Heppner, J. P., Polar cap electric field distributions related to the interplanetary magnetic field direction, J. Geophys. Res., 77, 4877-4887, 1972.

Heppner J. P., High latitude electric fields and the modulations related to interplanetary magnetic field parameters, Radio Sci., 8, 933-948, 1973.

Heppner, J. P., Empirical models of high-latitude electric fields, J. Geophys. Res., 82, 1115-1125, 1977.

Kamide, Y., A. D. Richmond, and S. Matsushita, Estimation of ionospheric electric fields, ionospheric currents, and field-aligned currents from ground magnetic records, J. Geophys. Res., 86, 801-813, 1981.

Kivelson, M. G., Magnetospheric electric fields and their variation with geomagnetic activity, Rev. Geophys., 14, 189-197, 1976.
Lyons, L. R., A simple model for polar cap convection patterns a generation of $\theta$ auroras, J. Geophys. Res., 90, 1561-1567, 1985 . Piddington, J. H., A hydromagnetic theory of geomagnetic storms and
auroras, Planet. Space Sci., 9, 947-957, 1962.

Potemra, T. A., L. J. Zanetti, P. F. Bythrow, and A. T. Y. Lui, Br dependent convection patterns during northward interplanetary
magnetic field, J. Geophys. Res., 89, 9753-9760, 1984 . magnetic field, J. Geophys. Res., 89, 9753-9760, 1984.

Reiff, P. H., and J. L. Burch, IMF $B_{y}$-dependent plasma flow and Birkeland currents in the dayside magnetosphere, 2, A global model for northward and southward IMF, J. Geophys. Res., 90, 1595-1609, 1985.

Reiff, P. H., R. W. Spiro, and T. W. Hill, Dependence of polar cap potential drop on interplanetary parameters, J. Geophys. Res., 86, 7639-7648, 1981.

Roble, R. G., R. E. Dickinson, and E. C. Ridley, Global circulation and temperature structure of the thermosphere with high-latitude plasma convection, J. Geophys. Res., 87, 1599-1614, 1982.

Schunk, R. W., and J. C. G. Walker, Theoretical ion densities in the lower ionosphere, Planet. Space Sci., 21, 1875-1896, 1973.

Schunk, R. W., and W. J. Raitt, Atomic nitrogen and oxygen ions in the daytime high-latitude $F$ region, J. Geophys. Res., 85, 1255-1272, 1980.

Sojka, J. J., and R. W. Schunk, A theoretical study of the high latitude $F$ region's response to magnetospheric storm inputs, J. Geophys. Res., $88,2112-2122,1983$.

Sojka, J. J., and R. W. Schunk, A theoretical study of the global $F$ region for June solstice, solar maximum, and low magnetic activity, J. Geophys. Res., 90, 5285-5298, 1985.

Sojka, J. J., W. J. Raitt, and R. W. Schunk, Effect of displaced geomag. netic and geographic poles on high-latitude plasma convection and ionospheric depletions, J. Geophys. Res., 84, 5943-5951, 1979.

Sojka, J. J., J. C. Foster, W. J. Raitt, R. W. Schunk, and J. R. Doupnik, High-latitude convection: Comparison of a simple model with incoherent scatter observations, J. Geophys. Res., 85, 703-709, 1980.

Sojka, J. J., W. J. Raitt, and R. W. Schunk, A theoretical study of the high-latitude winter $F$ region at solar minimum for low magnetic activity, J. Geophys. Res., 86, 609-621, 1981.

Spiro, R. W., R. A. Heelis, and W. B. Hanson, Ion convection and the formation of the mid-latitude $F$ region ionization trough, J. Geophys. Res., 83, 4255-4264, 1978.

Spiro, R. W., M. Harel, R. A. Wolf, and P. H. Reiff, Quantitative simulation of a magnetospheric substorm, 3, Plasmaspheric electric fields and evolution of the plasmapause, J. Geophys. Res., 86, 2261-2272, 1981.

Vasyliunas, V. M., Mathematical models of magnetospheric convection and its coupling to the ionosphere, in Particles and Fields in the Magnetosphere, edited by B. M. McCormac, p. 60, D. Reidel, Hingham, Mass., 1972.

Volland, H., A semiempirical model of large-scale magnetospheric electric fields, J. Geophys. Res., 78, 171-180, 1973.

Volland, H., Models of global electric fields within the magnetosphere, Ann. Geophys., 31, 154-174, 1975.

Volland, H., A model of the magnetospheric electric convection field, J. Geophys. Res., 83, 2695-2699, 1978.

Yasuhara, F., R. Greenwald, and S.-I. Akasofu, On the rotation of the polar cap potential pattern and associated polar phenomena, J. Geophys. Res., 88, 5773-5777, 1983.

C. E. Rasmussen, R. W. Schunk, and J. J. Sojka, Center for Atmospheric and Space Sciences, UMC 3400, Utah State University, Logan, UT 84322.

(Received December 2, 1985; revised April 2, 1986; accepted April 24, 1986.) 